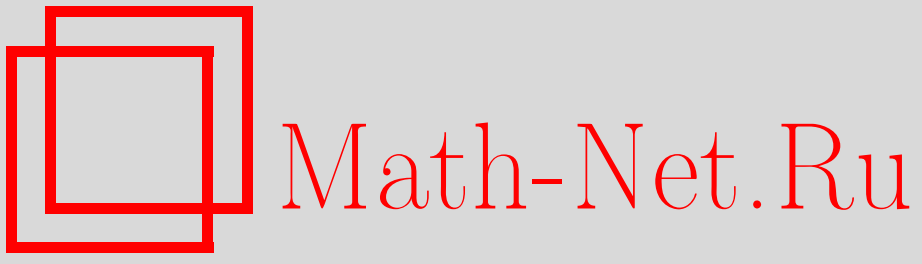

S. Graversen, А. Н. Ширяев, М. Йор, К вопросу о стохастических интегральных представлениях функционалов от броуновского движения. II, Теория вероятн. и ее примен., 2006, том 51, выпуск 1, 64-77

DOI: https://doi.org/10.4213/tvp146

Использование Общероссийского математического портала MathNet.Ru подразумевает, что вы прочитали и согласны с пользовательским соглашением

http://www.mathnet.ru/rus/agreement

Параметры загрузки:

IP : 54.237 .59 .107

26 апреля 2023 г., 13:06:21

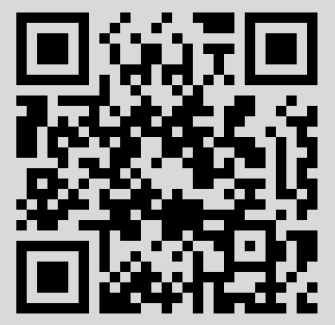


(C) 2006 г. ГРАВЕРСЕН С. Э.*, ШИРЯЕВ А. Н..* ЙОР М.***

\section{К ВОПРОСУ О СТОХАСТИЧЕСКИХ ИНТЕГРАЛЬНЫХ ПРЕДСТАВЛЕНИЯХ ФУНКЦИОНАЛОВ ОТ БРОУНОВСКОГО ДВИЖЕНИЯ. ІІ ${ }^{1)}$}

В первой части работы, [1], был изложен метод получения стохастических интегральных представлений для функционалов $S(\omega)$ от броуновского движения $B=\left(B_{t}\right)_{t \geqslant 0}$. В качестве иллюстрации были рассмотрены функционалы $\max _{t \leqslant T} B_{t}$ и $\max _{t \leqslant T_{-a}} B_{t}$, где $T_{-a}=\inf \left\{t: B_{t}=-a\right\}, a>0$. В настояшей работе будет дан иной вывод (С.Э. Граверсен; $\S 2^{*}, 3^{*}$ ) представлений для этих функционалов и даны два доказательства (§ 4) представления для функционала $\max _{t \leqslant g_{T}} B_{t}$, где (немарковский момент) $g_{T}=\sup \left\{0 \leqslant t \leqslant T: B_{t}=0\right\}$.

Ключевые слова и фразы: броуновское движение, интегралы Ито, тах-функционалы, стохастическое интегральное представление.

\section{$\S 2 *$ Второй вывод представления для $S_{T}=\max _{t \leqslant T} B_{t}$}

1. Согласно формуле (4) первой части работы,

$$
S_{T}=\mathbf{E} S_{T}+2 \int_{0}^{T}\left[1-\Phi\left(\frac{S_{t}-B_{t}}{\sqrt{T-t}}\right)\right] d B_{t}
$$

или, равносильно,

$$
S_{T}=\mathbf{E} S_{T}+\int_{0}^{T} \Psi\left(\frac{S_{t}-B_{t}}{\sqrt{T-t}}\right) d B_{t}
$$

где $S_{t}=\max _{u \leqslant t} B_{u}, \mathbf{E} S_{t}=\sqrt{2 T / \pi}$ и $\Psi(x)=2[1-\Phi(x)](=2 \mathbf{P}\{\mathscr{N}(0,1)>$ $x\}, \mathscr{N}(0,1)$ - стандартная гауссовская случайная величина).

2. Покажем, что для всякого $t \geqslant 0$ справедлива следуюшая формула:

$$
\mathbf{E}\left(S_{T} \mid \mathscr{F}_{t}\right)=\sqrt{\frac{2}{\pi} T}+\int_{0}^{T \wedge t} \Psi\left(\frac{S_{u}-B_{u}}{\sqrt{T-u}}\right) d B_{u},
$$

* Institute for Mathematical Sciences, Ny Munkegade, 8000 Aarhus C, Denmark; e-mail: matseg@imf.au.dk

** Математический институт им. В. А. Стеклова РАН, ул. Губкина, 8, 119991 Москва, ГСП-1, Россия; e-mail: albertsh@mi.ras.ru

*** Laboratoire de Probabilités et Modèles Aléatoires, Université Pierre et Marie Curie, Boîte courrier 188, 75252 Paris Cedex 05, France; e-mail: deaproba@proba.jussieu.fr

1) Второй автор поддержан РФФИ (грант 05-01-00944) и грантом НШ-1758.2003.1. 
из которой, очевидно, следует и формула (45). (Напомним, что $\mathscr{F}_{t}=$ $\sigma\left(B_{s}, s \leqslant t\right)$ есть $\sigma$-алгебра, порожденная броуновским движением и пополненная множествами нулевой $\mathbf{P}$-вероятности из $\sigma$-алгебры $\mathscr{F}$ исходного полного вероятностного пространства $(\Omega, \mathscr{F}, \mathbf{P})$.)

Зафиксируем $0 \leqslant t<T$. Тогда, согласно (5),

$$
\begin{aligned}
\mathbf{E}\left[S_{T} \mid \mathscr{F}_{t}\right] & =\mathbf{E}\left(\int_{0}^{\infty} \mathbf{E}\left[I\left(a<S_{t}\right) \mid \mathscr{F}_{t}\right] d a\right)=\int_{0}^{\infty} \mathbf{E}\left[I\left(T_{a}<T\right) \mid \mathscr{F}_{t}\right] d a \\
& =\int_{0}^{\infty}\left(I\left(T_{a} \leqslant t\right)+\mathbf{E}\left[I\left(t<T_{a}<T\right) \mid \mathscr{F}_{t}\right]\right) d a \\
& =\int_{0}^{\infty} I\left(T_{a} \leqslant t\right) d a+\int_{0}^{\infty} \mathbf{P}\left(t<T_{a}<T \mid \mathscr{F}_{t}\right) d a \\
& =S_{t}+\int_{0}^{\infty} \mathbf{P}\left(t<T_{a}<T \mid \mathscr{F}_{t}\right) d a
\end{aligned}
$$

На множестве $\left\{t<T_{a}\right\}$, согласно марковскому свойству и формулам (14), (15), мы имеем

$$
\begin{aligned}
\mathbf{P}\left(t<T_{a}<T \mid \mathscr{F}_{t}\right) & =\mathbf{P}\left(\exists s \in(t, T): B_{s}>a \mid \mathscr{F}_{t}\right) \\
& =\mathbf{P}_{B_{t}}\left\{\exists s \in(0, T-t): B_{s}>a\right\}=\mathbf{P}_{B_{t}}\left\{T_{a}<T-t\right\} \\
& =\int_{0}^{T-t} \frac{a-B_{t}}{\sqrt{2 \pi s^{3}}} \exp \left\{-\frac{\left(a-B_{t}\right)^{2}}{2 s}\right\} d s
\end{aligned}
$$

где $\mathbf{P}_{x}(\cdot)$ - распределение броуновского движения, начинающегося в точке $x$.

Из (47) и (48) находим, что

$$
\begin{aligned}
\mathbf{E}\left[S_{T} \mid \mathscr{F}_{t}\right] & =S_{t}+\int_{S_{t}}^{\infty} \int_{0}^{T-t} \frac{a-B_{t}}{\sqrt{2 \pi s^{3}}} \exp \left\{-\frac{\left(a-B_{t}\right)^{2}}{2 s}\right\} d s d a \\
& =S_{t}+\int_{0}^{T-t} \frac{1}{\sqrt{2 \pi s}}\left(\int_{S_{t}}^{\infty} \frac{a-B_{t}}{\sqrt{2 \pi s^{2}}} \exp \left\{-\frac{\left(a-B_{t}\right)^{2}}{2 s}\right\} d a\right) d s \\
& =S_{t}+\int_{0}^{T-t} \frac{1}{\sqrt{2 \pi s}} \exp \left\{-\frac{\left(S_{t}-B_{t}\right)^{2}}{2 s}\right\} d s \\
& =S_{t}+H\left(S_{t}-B_{t}, t\right)
\end{aligned}
$$

где

$$
H(x, t)=\int_{0}^{T-t} \frac{1}{\sqrt{2 \pi s}} e^{-x^{2} /(2 s)} d s, \quad x \in \mathbf{R}, \quad 0 \leqslant t<T .
$$

Ясно, что

$$
H(0,0)=\int_{0}^{T} \frac{1}{\sqrt{2 \pi s}} d s=\sqrt{\frac{2 T}{\pi}}
$$


и для $x>0$ и $0<t<T$

$$
\begin{aligned}
\frac{\partial}{\partial x} H(x, t) & =-\int_{0}^{T-t} \frac{x}{\sqrt{2 \pi s}} e^{-x^{2} /(2 s)} d s \\
& =-\mathbf{P}\left\{T_{x}<T-t\right\}=-\Psi\left(\frac{x}{\sqrt{T-t}}\right) .
\end{aligned}
$$

Обозначая $X_{t}=S_{t}-B_{t}$ и применяя к $H\left(X_{t}, t\right)$ формулу Ито, находим, что (в дифференциальной форме)

$$
d H\left(X_{t}, t\right)=\frac{\partial}{\partial t} H\left(X_{t}, t\right) d t+\frac{\partial}{\partial x} H\left(X_{t}, t\right) d X_{t}+\frac{1}{2} \frac{\partial^{2}}{\partial x^{2}} H\left(X_{t}, t\right) d[X]_{t},
$$

где $\left(\left[X_{t}\right]\right)_{t \leqslant T}-$ квадратическая вариация процесса $\left(X_{t}\right)_{t \leqslant T}$. Очевидно, что в рассматриваемом случае $[X]_{t}=[B]_{t}=t$. Поэтому (в интегральной форме)

$$
\begin{aligned}
H\left(S_{t}-B_{t}, t\right)=H(0,0)+ & {\left[\int_{0}^{t} \frac{\partial}{\partial u} H\left(X_{u}, u\right) d u+\int_{0}^{t} \frac{\partial}{\partial x} H\left(X_{u}, u\right) d S_{u}\right.} \\
& \left.+\frac{1}{2} \int_{0}^{t} \frac{\partial^{2}}{\partial x^{2}} H\left(X_{u}, u\right) d u\right]-\int_{0}^{t} \frac{\partial}{\partial x} H\left(X_{u}, u\right) d B_{u} .
\end{aligned}
$$

Обозначая выражение в квадратных скобках через $A_{t}$, находим из (49), что

$$
\mathbf{E}\left(S_{T} \mid \mathscr{F}_{t}\right)=H(0,0)+\left(A_{t}+B_{t}\right)-\int_{0}^{t} \frac{\partial}{\partial x} H\left(X_{u}, u\right) d B_{u}
$$

Поскольку процессы $\left(\mathbf{E}\left(S_{T} \mid \mathscr{F}_{t}\right)\right)_{t \leqslant T}$ и $\left(\int_{0}^{t} \frac{\partial}{\partial x} H\left(X_{u}, u\right) d B_{u}\right)_{t \leqslant T}$ являются непрерывными мартингалами, то процесс ограниченной вариации $\left(A_{t}+B_{t}\right)_{t \leqslant T}$ с $A_{0}+B_{0}=0$ также есть мартингал. Следовательно, этот процесс тождественно (с точностью до стохастической неразличимости) равен нулю, а значит,

$$
\mathbf{E}\left(S_{T} \mid \mathscr{F}_{t}\right)=H(0,0)-\int_{0}^{t} \frac{\partial}{\partial x} H\left(X_{u}, u\right) d B_{u},
$$

что с учетом формул (50) и (51) приводит к требуемому представлению (46).

§ 3*. Второй вывод представления для $S_{T_{-a}}=\max _{t \leqslant T_{-a}} B_{t}$

1. Согласно формуле (44) из первой части работы,

$$
S_{T_{-a}}=\int_{0}^{T_{-a}} \ln \frac{a}{a+S_{u}} d B_{u} .
$$

Покажем, что для каждого $M>0$ и $t \geqslant 0$ справедливо представление

$$
\mathbf{E}\left[S_{T_{-\mathrm{a}}} \wedge M \mid \mathscr{F}_{t}\right]=a \ln \frac{a+M}{a}+\int_{0}^{t \wedge T_{-\mathrm{a}}} \ln \frac{a+M}{a+M \wedge S_{u}} d B_{u},
$$


из которого, как будет показано ниже, предельным переходом по $M \rightarrow \infty$ получается и формула (52).

Для фиксированного $M>0$

$$
\begin{aligned}
\mathbf{E} & {\left[S_{T_{-a}} \wedge M \mid \mathscr{F}_{t}\right]=\mathbf{E}\left[\int_{0}^{M} I\left(\alpha<S_{T_{-a}} \wedge M\right) d \alpha \mid \mathscr{F}_{t}\right] } \\
= & \int_{0}^{M} \mathbf{E}\left[I\left(\alpha<S_{T_{-a} \wedge M}\right) \mid \mathscr{F}_{t}\right] d \alpha=\int_{0}^{M} \mathbf{E}\left[I\left(T_{\alpha}<T_{-a}\right) \mid \mathscr{F}_{t}\right] d \alpha \\
= & \int_{0}^{M} I\left(T_{\alpha}<T_{-a} \leqslant t\right) d \alpha \\
& +\int_{0}^{M}\left(I\left(T_{\alpha}<T_{-a}\right)+\mathbf{E}\left[I\left(t<T_{\alpha}<T_{-a}\right) \mid \mathscr{F}_{t}\right]\right) d \alpha .
\end{aligned}
$$

В силу марковского свойства броуновского движения находим, что на множестве $\left\{t<T_{-a}\right\}$

$$
\begin{aligned}
& \mathbf{E}\left[S_{T_{-a}} \wedge M \mid \mathscr{F}_{t}\right]=\int_{0}^{M} I\left(T_{\alpha} \leqslant t\right) d \alpha+\int_{0}^{M} \mathbf{E}\left[I\left(t<T_{\alpha}<T_{-a}\right) \mid \mathscr{F}_{t}\right] d \alpha \\
& =\int_{0}^{M} I\left(\alpha<S_{t}\right) d \alpha+\int_{0}^{M} \mathbf{E}\left[I\left(t<T_{\alpha}\right) I\left(0<T_{\alpha} \circ \theta_{t}<T_{-a} \circ \theta_{t}\right) \mid \mathscr{F}_{t}\right] d \alpha \\
& =S_{t} \wedge M+\int_{0}^{M} \mathbf{P}_{B_{t}}\left\{T_{\alpha}<T_{-a}\right\} I\left(t<T_{\alpha}\right) d \alpha,
\end{aligned}
$$

где $\theta_{t}$ - оператор сдвига. На множестве же $\left\{T_{-a} \leqslant t\right\}$

$$
\mathbf{E}\left[S_{T_{-a}} \wedge M \mid \mathscr{F}_{t}\right]=\int_{0}^{M} I\left(T_{\alpha}<T_{-a}<t\right) d \alpha=S_{T_{-a}} \wedge M .
$$

Воспользовавшись известной формулой

$$
\mathbf{P}_{x}\left\{T_{\alpha}<T_{-a}\right\}=\frac{x+a}{\alpha+a}, \quad-a<x<\alpha,
$$

из (55) находим, что на множестве $\left\{t<T_{-a}\right\}$

$$
\begin{aligned}
& \mathbf{E}\left[S_{T_{-a}} \wedge M \mid \mathscr{F}_{t}\right]=S_{t} \wedge M+\int_{0}^{M} \frac{B_{t}+a}{\alpha+a} I\left(t<T_{\alpha}\right) d \alpha \\
& \quad=S_{t} \wedge M+B_{t} \int_{0}^{M} \frac{1}{\alpha+a} I\left(S_{t}<\alpha\right) d \alpha+\int_{0}^{M} \frac{a}{\alpha+a} I\left(S_{t}<\alpha\right) d \alpha \\
& \quad=S_{t} \wedge M+\left(B_{t}+a\right) \ln (M+a)-B_{t} \ln \left(S_{t} \wedge M+a\right)-a \ln \left(S_{t} \wedge M+a\right) \\
& =A_{t}+\int_{0}^{t} \ln \frac{M+a}{S_{u} \wedge M+a} d B_{u}
\end{aligned}
$$

где $\left(A_{t}\right)_{t \geqslant 0}$ - непрерывный процесс ограниченной вариации, задаваемый формулой

$$
\begin{aligned}
A_{t}= & a \ln (M+a)+S_{t} \wedge M-a \ln \left(S_{t} \wedge M+a\right) \\
& -\int_{0}^{t} \frac{B_{\alpha}}{S_{u} \wedge M+a} I\left(S_{u} \leqslant M\right) d S_{u} .
\end{aligned}
$$


(При получении последнего равенства в (57) использована формула Ито, примененная к $B_{t} \ln \left(S_{t} \wedge M+a\right)$.)

Как и в конце $\S 2^{*}$, воспользуемся тем, что непрерывный мартингал, являющийся процессом ограниченной вариации, есть константа. Тогда из (57) находим, что процессы

$$
\left(\mathbf{E}\left[S_{T_{-a}} \wedge M \mid \mathscr{F}_{t \wedge T_{-a}}\right]\right)_{t \geqslant 0} \quad \text { и } \quad\left(A_{0}+\int_{0}^{t \wedge T_{-a}} \ln \frac{M+a}{S_{u} \wedge M+a} d B_{u}\right)_{t \geqslant 0}
$$

неразличимы.

Из (58) получаем равенство $A_{0}=a \ln [(M+a) / a]$, и, следовательно, для каждого $t \geqslant 0$

$$
\mathbf{E}\left(S_{T_{-a}} \wedge M \mid \mathscr{F}_{t}\right)=a \ln \frac{M+a}{a}+\int_{0}^{t \wedge T_{-a}} \ln \frac{M+a}{S_{u} \wedge M+a} d B_{u} \quad \text { (Р-п.н.), }
$$

что и есть требуемая формула (53).

Заметим, что согласно этой формуле

$$
\mathbf{E}\left(S_{T_{-a}} \wedge M\right)=a \ln \frac{M+a}{a} .
$$

Эту последнюю формулу можно было бы найти и непосредственно:

$$
\begin{aligned}
\mathbf{E}\left(S_{T_{-a}} \wedge M\right) & =\int_{0}^{M} \mathbf{P}\left\{S_{T_{-a}} \wedge M>\alpha\right\} d \alpha \\
& =\int_{0}^{M} \mathbf{P}\left\{T_{\alpha}<T_{-a}\right\} d \alpha=\int_{0}^{M} \frac{a}{\alpha+a} d \alpha=a \ln \frac{M+a}{a} .
\end{aligned}
$$

Если в (53) положить $t=T_{-a}$, то найдем, что для каждого $M>0$

$$
\begin{aligned}
S_{T_{-a}} \wedge M & =a \ln \frac{M+a}{a}+\int_{0}^{T_{-a}}\left[\ln (M+a)-\ln \left(S_{u} \wedge M+a\right)\right] d B_{u} \\
& =a \ln \frac{M+a}{a}-a \ln (M+a)-\int_{0}^{T_{-a}} \ln \left(S_{u} \wedge M+a\right) d B_{u} \\
& =\int_{0}^{T_{-a}}\left[\ln a-\ln \left(S_{u} \wedge M+a\right)\right] d B_{u}=\int_{0}^{T_{-a}} \ln \frac{a}{S_{u} \wedge M+a} d B_{u} .
\end{aligned}
$$

Полагая здесь $M \rightarrow \infty$ и используя непрерывность интеграла по $M$, находим

$$
S_{T_{-a}}=\int_{0}^{T_{-a}} \ln \frac{a}{a+S_{u}} d B_{u}
$$

что и есть требуемая формула (52).

2. Пусть

$$
T_{-a}^{b}=T_{b} \wedge T_{-a}, \quad a, b>0 .
$$

Иначе говоря, пусть

$$
T_{-a}^{b}=\inf \left\{t>0: B_{t} \notin(-a, b)\right\} .
$$


Рассуждения, сходные с приведенными, позволяют установить справедливость следующих представлений:

$$
S_{T_{-a}^{b}}=a \ln \frac{a+b}{a}+\int_{0}^{T_{-a}^{b}} \ln \frac{b+a}{S_{u}+a} d B_{u}
$$

и

$$
\mathbf{E}\left(S_{T_{-a}^{b}} \mid \mathscr{F}_{t}\right)=a \ln \frac{a+b}{a}+\int_{0}^{t \wedge T_{-a}^{b}} \ln \frac{b+a}{S_{u}+a} d B_{u} .
$$

Действительно, поскольку $\mathbf{P}\left\{S_{T_{-a}^{b}} \leqslant b\right\}=1$ и

$$
\mathbf{P}\left\{S_{T_{-a}^{b}}>u\right\}=\mathbf{P}\left\{T_{u}<T_{-a}\right\}=\frac{a}{u+a}, \quad 0<u \leqslant b,
$$

то видим, что

$$
\mathbf{E} S_{T_{-a}^{b}}=a \ln \frac{b+a}{a} .
$$

Зафиксируем $t>0$. Как и в п. 1 (см. (54)), имеем, что

$$
\begin{aligned}
\mathbf{E}\left[S_{T_{-a}^{b}} \mid \mathscr{F}_{t}\right]= & \int_{0}^{\infty} \mathbf{E}\left[I\left(u<S_{T_{-a}^{b}}\right) \mid \mathscr{F}_{t}\right] d u=\int_{0}^{\infty} \mathbf{E}\left[I\left(T_{u}<T_{-a}^{b}\right) \mid \mathscr{F}_{t}\right] d u \\
= & \int_{0}^{\infty}\left(I\left(T_{u}<T_{-a}^{b} \leqslant t\right)+I\left(T_{u} \leqslant t<T_{-a}^{b}\right)\right. \\
& \left.\quad+\mathbf{E}\left[I\left(t<T_{u}<T_{-a}^{b}\right) \mid \mathscr{F}_{t}\right]\right) d u .
\end{aligned}
$$

Используя марковское свойство броуновского движения, находим, что

$$
\begin{aligned}
\mathbf{E}\left[I\left(t<T_{u}<T_{-a}^{b}\right) \mid \mathscr{F}_{t}\right] & =\mathbf{P}\left(\exists s \in\left(t, T_{-a}^{b}\right): B_{s}>u \mid \mathscr{F}_{t}\right) I\left(t<T_{-a}^{b}\right) \\
& =\mathbf{P}_{B_{t}}\left\{\exists s \in\left(0, T_{-a}^{b}\right): B_{s}>u\right\} I\left(t<T_{-a}^{b}\right) \\
& =\psi\left(B_{t}, u\right) I\left(t<T_{-a}^{b}\right),
\end{aligned}
$$

где

$$
\psi(x, u)= \begin{cases}1, & x \geqslant u, u \in(0, b), \\ \frac{x+a}{u+a}, & -a<x<u, u \in(0, b), \\ 0, & \text { в других случаях. }\end{cases}
$$

С учетом этого обозначения находим, что на множестве $\left\{t<T_{-a}^{b}\right\}$

$$
\begin{aligned}
\mathbf{E}\left[S_{T_{-a}^{b}} \mid \mathscr{F}_{t}\right] & =S_{t}+\int_{S_{t}}^{b} \psi\left(B_{t}, u\right) d u \\
& =S_{t}+\int_{S_{t}}^{b} \frac{B_{t}+a}{u+a} d u=S_{t}+\left(B_{t}+a\right) \ln \frac{b+a}{S_{t}+a} .
\end{aligned}
$$

Применяя формулу Ито к правой части этого соотношения и снова, как и в п. 1, игнорируя члены ограниченной вариации, приходим к соотношению

$$
\mathbf{E}\left[S_{T_{-a}^{b}} \mid \mathscr{F}_{t}\right]=a \ln \frac{a+b}{a}+\int_{0}^{t \wedge T_{-a}^{b}} \ln \frac{b+a}{S_{u}+a} d B_{u},
$$


что и есть требуемая формула (60), из которой очевидным образом следует формула (59).

\section{§4. Случай $S_{g_{T}}=\max _{t \leqslant g_{T}} B_{t}$}

1. Пусть $g_{T}=\sup \left\{0<t \leqslant T: B_{t}=0\right\}$ есть момент последнего нуля броуновского движения на $(0, T]$. Если $B_{t} \neq 0$ для всех $0<t \leqslant T$, то полагаем $g_{T}=0$.

Теорема 3. Для $S_{g_{T}}$ справедливо следующее стохастическое интегральное представление:

$$
S_{g_{T}}=\frac{1}{2} \mathbf{E} S_{T}+\int_{0}^{T}\left[1-\Psi\left(\frac{2 S_{u}-B_{u}}{\sqrt{T-u}}\right)-Z_{u}\left(B_{u}, S_{u}-S_{g_{u}}\right)\right] d B_{u},
$$

где $\mathbf{E} S_{t}=\sqrt{2 T / \pi}, \Psi(x)=2[1-\Phi(x)] u$

$Z_{u}\left(B_{u}, S_{u}-S_{g_{u}}\right)=\left(S_{u}-S_{g_{u}}\right) \varphi_{T-u}\left(B_{u}\right) \quad c \quad g_{u}=\sup \left\{0<t \leqslant u: B_{t}=0\right\}$ или, равносильно,

$$
S_{g_{T}}=\frac{1}{2} \mathbf{E} S_{T}+\int_{0}^{T}\left[\frac{1}{2} \Psi\left(\frac{2 S_{u}-B_{u}}{\sqrt{T-u}}\right)-Z_{u}\left(B_{u}, S_{u}-S_{g_{u}}\right)\right] d B_{u} .
$$

Мы приводим два разных доказательства, каждое из которых может представлять самостоятельный интерес используемыми техническими средствами.

2. П ерв ое доказательст во. Имеем

$$
S_{g_{T}}=\int_{0}^{\infty} I\left(a<S_{g_{T}}\right) d a=\int_{0}^{\infty} I\left(g_{T}>T_{a}\right) d a=\int_{0}^{\infty} I\left(d_{T_{a}}<T\right) d a,
$$

где для $K>0$

$$
d_{K}=\inf \left\{t>K: B_{t}=0\right\} .
$$

По аналогии со схемой доказательства теоремы 1 естественно получить сначала стохастическое интегральное представление для $I\left(d_{T_{a}}<T\right)$ (ср. с леммой 1 , дающей представление для $I\left(T_{a}<T\right)$ ).

Лемма 4. Для всякого $а>0$ и всякого $T>0$

$$
\begin{aligned}
I\left(d_{T_{a}}<T\right)=\mathbf{P} & \left\{T_{2 a}<T\right\}+2 \int_{T_{a} \wedge T}^{d_{T_{a}} \wedge T} \varphi_{T-s}\left(B_{s}\right) d B_{s} \\
& -2 \int_{0}^{T_{a} \wedge T} \varphi_{T-s}\left(B_{s}-2 a\right) d B_{s} \quad(\mathbf{P}-\text { n.н. }) .
\end{aligned}
$$

Док а з те льст в о. Ясно, что

$$
\begin{aligned}
d_{T_{a}} & =\inf \left\{t>T_{a}: B_{t}=0\right\}=T_{a}+\inf \left\{u \geqslant 0: B_{T_{a}+u}=0\right\} \\
& =T_{a}+\inf \left\{u \geqslant 0: B_{T_{a}+u}-a=-a\right\} \\
& =T_{a}+\inf \left\{u \geqslant 0: \widehat{B}_{u}=-a\right\},
\end{aligned}
$$


где $\widehat{B}=\left(\widehat{B}_{u}\right)_{u \geqslant 0}$ с $\widehat{B}_{u}=B_{T_{a}+u}-a$ является броуновским движением, независимым от $\sigma$-алгебры $\mathscr{F}_{T_{a}}=\sigma\left\{A \in \mathscr{F}: A \cap\left\{T_{a} \leqslant t\right\} \in \mathscr{F}_{t}, t>0\right\}$ c $\mathscr{F}=\bigvee_{t>0} \mathscr{F}_{t}$.

\section{Обозначим}

$$
\widehat{T}_{-a}=\inf \left\{u \geqslant 0: \widehat{B}_{u}=-a\right\} .
$$

Тогда из (65) имеем $d_{T_{a}}=T_{a}+\widehat{T}_{-a}$, и, следовательно,

$$
I\left(d_{T_{a}}<T\right)=I\left(\widehat{T}_{-a}<T-T_{a}\right) .
$$

Найдем представление для $I\left(\widehat{T}_{-a}<b\right)$. Если обозначить $\widehat{T}_{-a}=\widehat{T}_{-a}(\widehat{B})$, то видим, что $\widehat{T}_{-a}(\widehat{B})=\widehat{T}_{a}(-\widehat{B})$. Согласно лемме 1 ,

$$
\begin{aligned}
I\left(\widehat{T}_{a}(-\widehat{B})<b\right) & =\mathbf{P}\left\{\widehat{T}_{a}(-\widehat{B})<b\right\}+2 \int_{0}^{\widehat{T}_{a}(-\widehat{B}) \wedge b} \varphi_{b-u}\left(-\widehat{B}_{u}-a\right) d\left(-\widehat{B}_{u}\right) \\
& =\mathbf{P}\left\{T_{a}<b\right\}-2 \int_{0}^{\widehat{T}_{-a} \wedge b} \varphi_{b-u}\left(\widehat{B}_{u}+a\right) d \widehat{B}_{u} .
\end{aligned}
$$

По формулам (14), (15)

$$
\mathbf{P}\left\{T_{a}<b\right\}=\int_{0}^{\infty} I(t<b) \gamma_{a}(t) d t \quad \text { с } \quad \gamma_{a}(t)=\frac{a}{\sqrt{2 \pi t^{3}}} e^{-a^{2} /(2 t)} .
$$

Следовательно,

$$
I\left(\widehat{T}_{-a}<b\right)=\int_{0}^{\infty} I(t<b) \gamma_{a}(t) d t-2 \int_{0}^{\widehat{T}_{-a} \wedge b} \varphi_{b-u}\left(\widehat{B}_{u}+a\right) d \widehat{B}_{u} .
$$

Пользуясь независимостью $\widehat{T}_{-a}$ от $\sigma$-алгебры $\mathscr{F}_{T_{a}}$, находим из (67), что

$$
\begin{aligned}
\int_{0}^{\widehat{T}_{-a} \wedge\left(T-T_{a}\right)} \varphi_{T-T_{a}-u}\left(\widehat{B}_{u}+a\right) d \widehat{B}_{u} & =\int_{0}^{\widehat{T}_{-a} \wedge\left(T-T_{a}\right)} \varphi_{T-T_{a}-u}\left(B_{T_{a}+u}\right) d B_{T_{a}+u} \\
=\int_{T_{a}}^{\left(\widehat{T}_{-a}+T_{a}\right) \wedge T} \varphi_{T-s}\left(B_{s}\right) d B_{s} & =\int_{T_{a} \wedge T}^{d_{T_{-a}} \wedge T} \varphi_{T-s}\left(B_{s}\right) d B_{s} .
\end{aligned}
$$

Отсюда и из (67), (66) и (6) получаем, что

$$
\begin{aligned}
I\left(d_{T_{a}}<T\right)= & \int_{0}^{T} I\left(T_{a}<T-t\right) \gamma_{a}(t) d t-2 \int_{T_{a} \wedge T}^{d_{T_{a}} \wedge T} \varphi_{T-s}\left(B_{s}\right) d B_{s} \\
= & \int_{0}^{T} \mathrm{P}\left\{T_{a}<T-t\right\} \gamma_{a}(t) d t \\
& +2 \int_{0}^{T}\left[\int_{0}^{T_{a} \wedge(T-t)} \varphi_{T-t-s}\left(B_{s}-a\right) d B_{s}\right] \gamma_{a}(t) d t \\
& -2 \int_{T_{a} \wedge T}^{d_{T_{a}} \wedge T} \varphi_{T-s}\left(B_{s}\right) d B_{s} \\
= & \mathbf{P}\left\{T_{2 a}<T\right\}+2 \int_{0}^{T_{a} \wedge T}\left[\int_{0}^{T-s} \gamma_{a}(t) \varphi_{T-t-s}\left(B_{s}-a\right) d t\right] d B_{s} \\
& -2 \int_{T_{a} \wedge T}^{d_{T_{a}} \wedge T} \varphi_{T-s}\left(B_{s}\right) d B_{s} .
\end{aligned}
$$


Согласно лемме 5, приводимой в п. 3 ниже, для всех $s<T_{a}$ таких, что $B_{s}<a$, выполнено равенство

$$
\int_{0}^{T-s} \gamma_{a}(t) \varphi_{T-t-s}\left(B_{s}-a\right) d t=\varphi_{T-s}\left(B_{s}-2 a\right) .
$$

Поэтому из (68) следует, что

$$
\begin{aligned}
I\left(d_{T_{a}}<T\right)= & \mathbf{P}\left\{T_{2 a}<T\right\}+2 \int_{0}^{T_{a} \wedge T} \varphi_{T-s}\left(B_{s}-2 a\right) d B_{s} \\
& -2 \int_{T_{a} \wedge T}^{d_{T_{a}} \wedge T} \varphi_{T-s}\left(B_{s}\right) d B_{s},
\end{aligned}
$$

что и есть требуемая формула (64). Лемма 4 доказана.

Обратимся теперь к доказательству представления (61).

В силу (63) и (64)

$$
\begin{aligned}
S_{g_{T}}= & \int_{0}^{\infty} I\left(d_{T_{a}}<T\right) d a \\
= & \int_{0}^{\infty} \mathbf{P}\left\{T_{2 a}<T\right\} d a+2 \int_{0}^{\infty}\left[\int_{0}^{T_{a} \wedge T} \varphi_{T-s}\left(B_{s}-2 a\right) d B_{s}\right] d a \\
& -2 \int_{0}^{\infty}\left[\int_{T_{a} \wedge T}^{d_{T_{a}} \wedge T} \varphi_{T-s}\left(B_{s}\right) d B_{s}\right] d a .
\end{aligned}
$$

Здесь

$$
\int_{0}^{\infty} \mathbf{P}\left\{T_{2 a}<T\right\} d a=\frac{1}{2} \int_{0}^{\infty} \mathbf{P}\left\{T_{b}<T\right\} d b=\frac{1}{2} \int_{0}^{\infty} \mathbf{P}\left\{S_{T}>b\right\} d b=\frac{1}{2} \mathbf{E} S_{T}
$$

и

$$
\begin{aligned}
\int_{0}^{\infty} & {\left[\int_{0}^{T_{a} \wedge T} \varphi_{T-s}\left(B_{s}-2 a\right) d B_{s}\right] d a } \\
= & \int_{0}^{T}\left[\int_{0}^{\infty} \varphi_{T-u}\left(B_{u}-2 a\right) I\left(S_{u}<a\right) d a\right] d B_{u} \\
= & \int_{0}^{T}\left[\int_{S_{u}}^{\infty} \varphi_{T-u}\left(B_{u}-2 a\right) d a\right] d B_{u}=\frac{1}{2} \int_{0}^{T}\left[\int_{2 S_{u}}^{\infty} \varphi_{T-u}\left(B_{u}-b\right) d b\right] d B_{u} \\
= & \frac{1}{2} \int_{0}^{T}\left[1-\Phi\left(\frac{2 S_{u}-B_{u}}{\sqrt{T-u}}\right)\right] d B_{u} .
\end{aligned}
$$

Наконец, преобразуем последнее выражение в правой части (70).

Имеем

$$
\begin{aligned}
\int_{0}^{\infty} & {\left[\int_{T_{a} \wedge T}^{d_{T_{a}} \wedge T} \varphi_{T-s}\left(B_{s}\right) d B_{s}\right] d a } \\
= & \int_{0}^{\infty} \int_{0}^{\infty} I\left(T_{a} \wedge T<s<d_{T_{a}} \wedge T\right) \varphi_{T-s}\left(B_{s}\right) d B_{s} d a \\
= & \int_{0}^{T}\left[\int_{0}^{\infty} I\left(T_{a}<s<d_{T_{a}} \wedge T\right) d a\right] \varphi_{T-s}\left(B_{s}\right) d B_{s}
\end{aligned}
$$




$$
\begin{aligned}
& =\int_{0}^{T}\left[\int_{0}^{\infty} I\left(S_{g_{s}}<a<S_{s}\right) d a\right] \varphi_{T-s}\left(B_{s}\right) d B_{s} \\
& =\int_{0}^{T}\left(S_{u}-S_{g_{u}}\right) \varphi_{T-u}\left(B_{u}\right) d B_{u} .
\end{aligned}
$$

Таким образом, из (70)-(73) следует, что

$$
\begin{aligned}
S_{g_{T}}= & \frac{1}{2} \mathbf{E} S_{T}+\int_{0}^{T}\left[1-\Phi\left(\frac{2 S_{u}-B_{u}}{\sqrt{T-u}}\right)\right] d B_{u} \\
& +2 \int_{0}^{T}\left(S_{u}-S_{g_{u}}\right) \varphi_{T-u}\left(B_{u}\right) d B_{u} .
\end{aligned}
$$

Тем самым формулы (61), (62) доказаны.

3. В приведенном выше доказательстве было использовано интегральное соотношение (69), связывающее плотности

$$
\varphi_{t}(a)=\frac{1}{\sqrt{2 \pi t}} e^{-a^{2} /(2 t)} \quad \text { и } \quad \gamma_{a}(t)=\frac{a}{\sqrt{2 \pi t^{3}}} e^{-a^{2} /(2 t)} \quad\left(=-\frac{\partial}{\partial a} \varphi_{t}(a)\right) .
$$

Вытекает оно из следующего предложения.

Лемма 5. Для всех $a>0 u \theta>0$

$$
\int_{0}^{\theta} \gamma_{a}(t) \varphi_{\theta-t}(x-a) d t= \begin{cases}\varphi_{\theta}(x), & x>a, \\ \varphi_{\theta}(x-2 a), & x \leqslant a .\end{cases}
$$

Д оказательст в о. Положим

$$
I(a, x)=\frac{1}{\varphi_{\theta}(x)} \int_{0}^{\theta} \gamma_{a}(t) \varphi_{\theta-t}(x-a) d t .
$$

Используя указанный выше явный вид функций $\varphi_{\theta}(a)$ и $\gamma_{a}(t)$ и делая замену переменных $u=\sqrt{\theta / t-1}$, находим, что

$$
I(a, x)=\frac{2 a}{\sqrt{2 \pi t}} e^{a(x-a) / \theta} \int_{0}^{\infty} e^{-\alpha u^{2}-\beta / u^{2}} d u
$$

с $\alpha=a^{2} /(2 \theta)$ и $\beta=(x-a)^{2} /(2 \theta)$.

По формуле 3.325 из [2]

$$
\int_{0}^{\infty} e^{-\alpha u^{2}-\beta / u^{2}} d u=\frac{1}{2} \sqrt{\frac{\pi}{\alpha}} e^{-2 \sqrt{\alpha \beta}}=\frac{\sqrt{2 \pi \theta}}{2 a} e^{-a|x-a| / \theta} .
$$

Следовательно,

$$
I(a, x)=e^{a(x-a) / \theta} e^{-a|x-a| / \theta}= \begin{cases}1, & x>a, \\ e^{-2 a(a-x) / \theta}, & x \leqslant a,\end{cases}
$$

что и доказывает требуемую формулу (74).

Наряду с приведенным «аналитическим» доказательством формулы (74) небезынтересно следующее «вероятностное» доказательство этой формулы. 
Пусть $f=f(x)$ - измеримая ограниченная функция. Тогда

$$
\mathbf{E} f\left(B_{\theta}\right)=\mathbf{E}\left[f\left(B_{\theta}\right) I\left(T_{a}<\theta\right)\right]+\mathbf{E}\left[f\left(B_{\theta}\right) I\left(T_{a} \geqslant \theta\right)\right] .
$$

Здесь

II

$$
\begin{aligned}
\mathbf{E}\left[f\left(B_{\theta}\right) I\left(T_{a}<\theta\right)\right] & =\mathbf{E}\left[f\left(B_{T_{a}+\left(\theta-T_{a}\right)}\right) I\left(T_{a}<\theta\right)\right] \\
& =\int_{0}^{\theta} \gamma_{a}(t)\left[\int_{-\infty}^{\infty} f(x) \varphi_{\theta-t}(x-a) d x\right] d t
\end{aligned}
$$

$$
\begin{aligned}
\mathbf{E}\left[f\left(B_{\theta}\right) I\left(T_{a} \geqslant \theta\right)\right] & =\int_{-\infty}^{\infty} \mathbf{E}\left[f\left(B_{\theta}\right) I\left(\theta \leqslant T_{a}\right) \mid B_{\theta}=x\right] \varphi_{\theta}(x) d x \\
& =\int_{-\infty}^{\infty} f(x) \mathbf{P}\left(T_{a} \geqslant \theta \mid B_{\theta}=x\right) \varphi_{\theta}(x) d x .
\end{aligned}
$$

Из (75)-(77) находим, что

$$
\begin{aligned}
\mathbf{E} f\left(B_{\theta}\right) & =\int_{-\infty}^{\infty} f(x) \varphi_{\theta}(x) d x \\
& =\int_{-\infty}^{\infty} f(x)\left[\int_{0}^{\theta} \varphi_{\theta-t}(x-a) \gamma_{a}(t) d t+\mathbf{P}\left(T_{a} \geqslant \theta \mid B_{\theta}=x\right) \varphi_{\theta}(x)\right] d x
\end{aligned}
$$

Отсюда в силу произвольности функции $f(x)$ получаем, что

$$
\int_{0}^{\theta} \gamma_{a}(t) \varphi_{\theta-t}(x-a) d t=\varphi_{\theta}(x)\left[1-\mathbf{P}\left(T_{a} \geqslant \theta \mid B_{\theta}=x\right)\right] .
$$

Если $x>a$, то $\mathrm{P}\left(T_{a} \geqslant \theta \mid B_{\theta}=x\right)=0$ и формула (78) дает (74).

Пусть теперь $x \leqslant a$, тогда

$$
\begin{aligned}
1-\mathbf{P}\left(T_{a} \geqslant \theta \mid B_{\theta}=x\right) & =\mathbf{P}\left(T_{a}<\theta \mid B_{\theta}=x\right)=\mathbf{P}\left(\max _{u \leqslant \theta} B_{u}>a \mid B_{\theta}=x\right) \\
& =\mathbf{P}\left(S_{\theta}>a \mid B_{\theta}=x\right)
\end{aligned}
$$

c $S_{\theta}=\max _{u \leqslant \theta} B_{u}$.

Вероятность $\mathrm{P}\left(S_{\theta}>a \mid B_{\theta}=x\right)$ можно найти, воспользовавшись, например, результатом Сешадри (см. [3]) о том, что величины $S_{\theta}\left(S_{\theta}-B_{\theta}\right)$ и $B_{\theta}$ независимы и

$$
S_{\theta}\left(S_{\theta}-B_{\theta}\right) \stackrel{\operatorname{law}}{=} \frac{\theta}{2} \mathscr{E}
$$

где $\mathscr{E}-$ стандартная экспоненциально распределенная случайная величина $\left(\mathbf{P}\{\mathscr{E}>t\}=e^{-t}, t>0\right)$.

Действительно, из сформулированных утверждений находим, что для $x \leqslant a, a \geqslant 0$ и $b=a(a-x)$

$$
\begin{aligned}
\mathbf{P}\left(S_{\theta}>a \mid B_{\theta}=x\right) & =\mathbf{P}\left(S_{\theta}\left(S_{\theta}-B_{\theta}\right)>b \mid B_{\theta}=x\right)=\mathbf{P}\left\{\frac{\theta}{2} \mathscr{E}>b\right\} \\
& =\mathbf{P}\left\{\mathscr{E}>\frac{2 b}{\theta}\right\}=e^{-2 b / \theta}=e^{-2 a(a-x) / \theta}
\end{aligned}
$$


Тем самым, входящее в (78) выражение $1-\mathbf{P}\left(T_{a} \geqslant \theta \mid B_{\theta}=x\right)$ равно

$$
\mathbf{P}\left(S_{\theta}>a \mid B_{\theta}=x\right)=e^{-2 a(a-x) / \theta}
$$

и, значит, для $x \leqslant a$

$$
\int_{0}^{\theta} \gamma_{a}(t) \varphi_{\theta-t}(x-a) d t=\varphi_{\theta}(x) e^{-2 a(a-x) / \theta},
$$

что и утверждается в (74).

4. В т о р о е д о к аз а т е л с с т в о. По аналогии с распределениями, использованными в $\S 2^{*}$ и $\S 3^{*}$, покажем, что для каждого $t>0$ справедливо (Р-п.н.) равенство

$$
\mathbf{E}\left[S_{g_{T}} \mid \mathscr{F}_{t}\right]=\frac{1}{2} \mathbf{E} S_{T}+\int_{0}^{t \wedge T}\left[\frac{1}{2} \Psi\left(\frac{2 S_{u}-B_{u}}{\sqrt{T-u}}\right)-Z_{u}\left(B_{u}, S_{u}-S_{g_{u}}\right)\right] d B_{u} .
$$

(Из этого представления следует, конечно, формула (61).)

Зафиксируем $0 \leqslant t<T$. Тогда

$$
\begin{gathered}
\mathbf{E}\left[S_{g_{T}} \mid \mathscr{F}_{t}\right]=\int_{0}^{\infty} \mathbf{E}\left[I\left(a<S_{g_{T}}\right) \mid \mathscr{F}_{t}\right] d a=\int_{0}^{\infty} \mathbf{E}\left[I\left(T_{a}<g_{T}\right) \mid \mathscr{F}_{t}\right] d a \\
=\int_{0}^{\infty} \mathbf{E}\left[I\left(T_{a}<g_{T} \leqslant t\right)+I\left(T_{a} \leqslant t<g_{T}\right)\right. \\
\left.\quad+I\left(t<T_{a}<g_{T}\right) \mid \mathscr{F}_{t}\right] d a .
\end{gathered}
$$

Воспользовавшись марковским свойством броуновского движения, находим следующие соотношения:

(a) $\mathbf{E}\left[I\left(T_{a}<g_{T} \leqslant t\right) \mid \mathscr{F}_{t}\right]=\mathbf{E}\left[I\left(T_{a}<g_{T}<t\right) \mid \mathscr{F}_{t}\right]$

$$
\begin{aligned}
= & \mathbf{E}\left[I\left(T_{a}<g_{T}<t\right) \mid \mathscr{F}_{t}\right] I\left(T_{a}<t\right) \\
= & \mathbf{P}\left(\exists s_{1}<s_{2}<t: B_{s_{1}}>a, B_{s_{2}}=0 ;\right. \\
& \left.\quad B_{s} \neq 0 \text { для } s \in(t, T) \mid \mathscr{F}_{t}\right) I\left(T_{a}<t\right) \\
= & \mathbf{P}\left(T_{a}+T_{0} \circ \theta_{T_{a}}<t \text { и } B_{s} \neq 0 \text { для } s \in(t, T) \mid \mathscr{F}_{t}\right) I\left(T_{a}<t\right) \\
= & \mathbf{P}_{B_{t}}\left\{B_{s} \neq 0 \text { для } s \in(0, T-t)\right\} I\left(T_{a}+T_{0} \circ \theta_{T_{a}}<t\right) \\
= & \mathbf{P}\left\{T_{\left|B_{t}\right|}>1-t\right\} I\left(T_{a}+T_{0} \circ \theta_{T_{a}}<t\right) ;
\end{aligned}
$$

(b) $\mathbf{E}\left[I\left(T_{a} \leqslant t \leqslant g_{T}\right) \mid \mathscr{F}_{t}\right]=\mathbf{E}\left[I\left(T_{a}<t<g_{T}\right) \mid \mathscr{F}_{t}\right]$

$$
\begin{aligned}
& =\mathbf{E}\left[I\left(T_{a}<t<g_{T}\right) \mid \mathscr{F}_{t}\right] I\left(t>T_{a}\right) \\
& =\mathbf{P}\left(\exists s<t: B_{s}>a \text { и } \exists s_{1} \in(t, T): B_{s_{1}}=0 \mid \mathscr{F}_{t}\right) I\left(t>T_{a}\right) \\
& =\mathbf{P}_{B_{t}}\left\{\exists s_{1} \in(0, T-t): B_{s_{1}}=0\right\} I\left(t>T_{a}\right) \\
& =\mathbf{P}\left\{T_{\left|B_{t}\right|}>T-t\right\} I\left(t>T_{a}\right) ;
\end{aligned}
$$


(c) $\mathbf{E}\left[I\left(t<T_{a}<g_{T}\right) \mid \mathscr{F}_{t}\right]=\mathbf{P}\left(\exists s_{1}, s_{2}: t<s_{1}<s_{2}<T\right.$,

$$
\begin{aligned}
& \left.B_{s_{1}}>a, B_{s_{2}}=0 \mid \mathscr{F}_{t}\right) I\left(t<T_{a}\right) \\
= & \mathbf{P}_{B_{t}}\left(\exists s_{1}, s_{2}: 0<s_{1}<s_{2}<T-t, B_{s_{1}}>a, B_{s_{2}}=0\right) I\left(t<T_{a}\right) \\
= & \mathbf{P}_{B_{t}}\left\{\exists s \in(0, T-t): B_{s}=2 a\right\} I\left(t<T_{a}\right) \\
= & \mathbf{P}\left\{T_{2 a-B_{t}}<T-t\right\} I\left(t<T_{a}\right) .
\end{aligned}
$$

Из (a), (b) и (c) получаем, что

$$
\begin{aligned}
& \begin{aligned}
\left(\mathrm{a}^{*}\right) \quad \int_{0}^{\infty} & \mathbf{E}\left[I\left(T_{a}<g_{T} \leqslant t\right) \mid \mathscr{F}_{t}\right] d a \\
\quad & \mathbf{P}\left\{T_{\left|B_{t}\right|}>T-t\right\} \int_{0}^{\infty} I\left(T_{a}+T_{0} \circ \theta_{T_{a}}<t\right) d a \\
& =\mathbf{P}\left\{T_{\left|B_{t}\right|}>T-t\right\} S_{g_{t}}=\left[1-\mathbf{P}\left\{T_{\left|B_{t}\right|}<T-t\right\}\right] S_{g_{t}}
\end{aligned} \\
& \left(\mathrm{~b}^{*}\right) \quad \int_{0}^{\infty} \mathbf{E}\left[I\left(T_{a}<t<g_{t}\right) \mid \mathscr{F}_{t}\right] d a=\mathbf{P}\left\{T_{\left|B_{t}\right|}<T-t\right\} S_{t} \\
& \left(\mathrm{c}^{*}\right) \quad \int_{0}^{\infty} \mathbf{E}\left[I\left(t<T_{a}<g_{T}\right) \mid \mathscr{F}_{t}\right] d a \\
& =\int_{0}^{\infty} \mathbf{P}\left\{T_{2 a-B_{t}}<T-t\right\} I\left(t<T_{a}\right) d a \\
& =\int_{S_{t}}^{\infty} \int_{0}^{T-t} \frac{2 a-B_{t}}{\sqrt{2 \pi s^{3}}} \exp \left\{-\frac{\left(2 a-B_{t}\right)^{2}}{2 s}\right\} d s d a \\
& =\int_{0}^{T-t} \int_{S_{t}}^{\infty} \frac{2 a-B_{t}}{\sqrt{2 \pi s^{3}}} \exp \left\{-\frac{\left(2 a-B_{t}\right)^{2}}{2 s}\right\} d a d s \\
& =\frac{1}{2} \int_{0}^{T-t} \frac{1}{\sqrt{2 \pi s}} \exp \left\{-\frac{\left(2 S_{t}-B_{t}\right)^{2}}{2 s}\right\} d s \\
& =\frac{1}{2} \int_{0}^{T-t} \varphi_{s}\left(2 S_{t}-B_{t}\right) d s=\frac{1}{2} H\left(2 S_{t}-B_{t}, t\right)
\end{aligned}
$$

где $H(x, t)=\int_{0}^{T-t} \varphi_{s}(x) d s$ и $0 \leqslant t<T$.

Собирая соотношения $\left(\mathrm{a}^{*}\right),\left(\mathrm{b}^{*}\right)$ и $\left(\mathrm{c}^{*}\right)$ вместе, получаем, что

$$
\begin{aligned}
\mathbf{E}\left(S_{g_{T}} \mid \mathscr{F}_{t}\right)= & \frac{1}{2} H\left(2 S_{t}-B_{t}, t\right)+\mathbf{P}\left\{T_{\left|B_{t}\right|}<T-t\right\}\left(S_{t}-S_{g_{t}}\right)+S_{g_{t}} \\
= & \frac{1}{2} H\left(2 S_{t}-B_{t}, t\right) \\
& +\int_{0}^{T-t} \frac{\left|B_{t}\right|}{\sqrt{2 \pi s^{3}}} \exp \left\{-\frac{\left|B_{t}\right|^{2}}{2 s}\right\} d s \cdot\left(S_{t}-S_{g_{t}}\right)+S_{g_{t}} .
\end{aligned}
$$

Применяя формулу Ито к $H\left(X_{t}, t\right)$ с $X_{t}=2 S_{t}-B_{t}$ (процесс Бесселя порядка 3), находим, что для $t<T$

$$
H\left(2 S_{t}-B_{t}, t\right)=H(0,0)-\int_{0}^{t} \frac{\partial}{\partial x} H\left(X_{u}, u\right) d B_{u}+A_{t}
$$


где $\left(A_{t}\right)_{t<T}$ - процесс ограниченной вариации. Отсюда в силу $(50)$ и $(51)$ получаем

$$
H\left(2 S_{t}-B_{t}, t\right)=\sqrt{\frac{2 T}{\pi}}+\int_{0}^{t} \Psi\left(\frac{2 S_{u}-B_{u}}{\sqrt{T-u}}\right) d B_{u}+A_{t}
$$

где $\left(A_{t}\right)_{t<T}$ - непрерывный процесс ограниченной вариации.

Положим

$$
\tilde{H}(x, t)=\int_{0}^{T-t} \frac{x}{\sqrt{2 \pi s^{3}}} e^{-x^{2} /(2 s)} d s \quad\left(=\Psi\left(\frac{x}{\sqrt{T-t}}\right)\right) .
$$

Применяя к $\tilde{H}\left(\left|B_{t}\right|, t\right)$ формулу Ито-Танака, находим, что

$$
\begin{aligned}
d \tilde{H}\left(\left|B_{t}\right|, t\right) & =\Psi\left(\frac{\left|B_{t}\right|}{\sqrt{T-t}}\right)=-2 \varphi\left(\frac{\left|B_{t}\right|}{\sqrt{T-t}}\right) \frac{1}{\sqrt{T-t}} \operatorname{sign} B_{t} d B_{t}+d \tilde{A}_{t} \\
& =-2 \varphi_{T-t}\left(B_{t}\right) \operatorname{sign} B_{t} d B_{t}+d \tilde{A}_{t}
\end{aligned}
$$

где $\left(\tilde{A}_{t}\right)_{t<T}-$ некоторый процесс ограниченной вариации.

Тем самым, из (81), (83)-(85) находим, пренебрегая членами ограниченной вариации (ср. с рассуждениями в конце $\S 2^{*}$ ), что для $t<T$

$$
\begin{aligned}
\mathbf{E}\left[S_{g_{T}} \mid \mathscr{F}_{t}\right]= & \sqrt{\frac{T}{2 \pi}}+\frac{1}{2} \int_{0}^{t \wedge T} \Psi\left(\frac{2 S_{u}-B_{u}}{\sqrt{T-t}}\right) d B_{u} \\
& -2 \int_{0}^{t \wedge T} \varphi_{T-u}\left(B_{u}\right) \operatorname{sign} B_{u} \cdot\left(S_{u}-S_{g_{u}}\right) d B_{u} .
\end{aligned}
$$

Заметим, что $\operatorname{sign} B_{u}$ здесь можно опустить, поскольку если $\operatorname{sign} B_{u}=-1$, то $S_{u}-S_{g_{u}}=0$. Значит,

$$
\int_{0}^{t \wedge T} \varphi_{T-u}\left(B_{u}\right) \operatorname{sign} B_{u} \cdot\left(S_{u}-S_{g_{u}}\right) d B_{u}=\int_{0}^{t \wedge T} \varphi_{T-u}\left(B_{u}\right)\left(S_{u}-S_{g_{u}}\right) d B_{u} .
$$

Требуемая формула (79) следует для $t<T$ из (86) и (87). В общем случае $($ когда $t \geqslant 0)$ надо лишь заметить, что $\lim _{t \uparrow T} \mathbf{E}\left[S_{g_{T}} \mid \mathscr{F}_{t}\right]=S_{g_{T}}$ и пределы $\lim _{t \uparrow T}$ интегралов $\int_{0}^{t \wedge T}(\cdot) d B_{u}$ в (86) равны интегралам $\int_{0}^{T}(\cdot) d B_{u}$.

\section{СПИСОК ЛИТЕРАТУРЫ}

1. Ширяев A. Н., Йор М. К вопросу о стохастических интегральных представлениях функционалов от броуновского движения. I. - Теория вероятн. и ее примен., 2003, т. 48 , в. 2 , c. $375-385$.

2. Градитейн И.С., Рыжих И.М. Таблицы интегралов, сумм, рядов и произведений. М.: Наука, 1971, 1108 c.

3. Seshadri V. Exponential models, Brownian motion, and independence. - Canad. J. Statist., 1988, v. 16, № 3, c. 209-221. 